\title{
CONVEX FUNCTIONS OF BOUNDED TYPE
}

\author{
A. W. GOODMAN
}

\begin{abstract}
We introduce a new class of normalized functions univalent and convex in the unit disk. These are called convex of bounded type and the set is denoted by $C V\left(R_{1}, R_{2}\right)$. For this set we find the Koebe domain, a coefficient bound, and a bound for $|f(z)|$. We also mention a few of the many questions that can be asked about this new class of univalent functions.
\end{abstract}

1. Introduction. Let $C V(\alpha)$ denote the set of functions that are convex of order $\alpha$. These are the functions of the form

$$
f(z)=z+\sum_{n=2}^{\infty} a_{n} z^{n}
$$

that are regular and univalent in the unit disk $E:|z|<1$, and for which

$$
\operatorname{Re} Q_{C V}(f) \equiv \operatorname{Re}\left(1+z \frac{f^{\prime \prime}(z)}{f^{\prime}(z)}\right)>\alpha, \quad z \text { in } E
$$

Here, of course, we must have $0 \leqslant \alpha \leqslant 1$ (see [ 3 and 1, vol. I, pp. 137-142]).

The class $C V(\alpha)$ has been studied extensively, but the geometric properties of $f(E)$ under a function in $C V(\alpha)$ are not immediately clear. The condition (1.2) states that the curve $C$ that bounds $f(E)$ satisfies the condition

$$
d \psi / d \theta \geqslant \alpha
$$

whenever this derivative exists. The difficulty lies in the fact that $\psi$ is an angle in the $w$-plane and $\theta$ is an angle in the $z$-plane. Thus the geometric implication of (1.3) is not obvious. Our purpose is to introduce and study a similar class of functions where the geometric nature of $f(E)$ is readily observable. Briefly we place upper and lower bounds on the curvature of $C$. It is more convenient to use $\rho$, the radius of curvature (the reciprocal of the curvature). By a formula due to Study [4], the radius of curvature of $f(|z|=r)$ is given by

$$
\rho=\frac{\left|z f^{\prime}(z)\right|}{\operatorname{Re} Q_{C V}(f)}, \quad z=r e^{i \theta} .
$$

Received by the editors July 7, 1983. Presented to the Society, January 1984, Louisville, Kentucky. 1980 Mathematics Subject Classification. Primary 30C45; Secondary 30C50.

Key words and phrases. Univalent functions, convex functions, coefficient bounds, Koebe domains. 
Briefly we ask that $R_{1} \leqslant \rho \leqslant R_{2}$. However, such a condition cannot be imposed throughout $E$ because $\rho \rightarrow 0$ as $z \rightarrow 0$, and our interest centers on the boundary of $E$. This forces a more complicated definition. Let

$$
\rho_{1}(r)=\min _{|z|=r} \rho \text { and } \rho_{2}(r)=\max _{|z|=r} \rho .
$$

Set

$$
R_{3}=\liminf \rho_{1} \quad \text { and } \quad R_{4}=\lim \sup \rho_{2}
$$

as $r \rightarrow 1^{-}$.

Definition 1. Let $R_{1}$ and $R_{2}$ be fixed in $[0, \infty]$. We say that $f(z)$ of the form (1), regular and univalent in $E$, is in the class $C V\left(R_{1}, R_{2}\right)$ if $R_{1} \leqslant R_{3}$ and $R_{4} \leqslant R_{2} . A$ function in $C V\left(R_{1}, R_{2}\right)$ with $0<R_{1} \leqslant R_{2}<\infty$ is said to be a convex function of bounded type.

Thus, by definition, the sets $C V\left(R_{1}, R_{2}\right)$ are increasing as either $R_{1} \rightarrow 0$ or $R_{2} \rightarrow \infty$, and the union over all $R_{1}, R_{2}$ is the set of all normalized convex functions. Let $C$ be the boundary of $f(E)$. Then by definition, if $f(z) \in C V\left(R_{1}, R_{2}\right)$, then on C

$$
R_{1} \leqslant d s / d \psi=\rho \leqslant R_{2}
$$

Here $s$ is arc length on $C$ and $\psi$ is the angle the tangent to $C$ makes with the positive real axis. Since both $s$ and $\psi$ are in the $w$-plane, the geometric character of $f(z)$ is clear.

If a simple closed curve satisfies the condition (1.7) with $0<R_{1} \leqslant R_{2}<\infty$, we will call it a convex curve of bounded type, and (by abuse of notation) we will write that $C \in C V\left(R_{1}, R_{2}\right)$. The investigation of such curves has a long history. Some useful results and further references may be found in [2].

Clearly the set of functions $C V\left(R_{1}, R_{2}\right)$ is invariant under the rotation $g(z)=$ $e^{-i \gamma} f\left(z e^{i \gamma}\right)$. Let $\overline{C V}\left(R_{1}, R_{2}\right)$ be the subset of $C V\left(R_{1}, R_{2}\right)$ for which the bounds $R_{1}$ and $R_{2}$ in (1.7) are actually attained on $C$. The transformation

$$
g(z)=\frac{f((z+a) /(1+\bar{a} z))-f(a)}{f^{\prime}(z)\left(1-|a|^{2}\right)}
$$

will take a function in $\overline{C V}\left(R_{1}, R_{2}\right)$ into a function in the same class, if and only if $\left|f^{\prime}(a)\left(1-|a|^{2}\right)\right|=1$. Thus (1.8) seems to be useless in the study of $C V\left(R_{1}, R_{2}\right)$.

In many studies of the set $S$ and its various subsets, the new function $g(z) \equiv$ $f(r z) / r$, with $0<r<1$, will belong to the same set as the primitive function $f(z)$ does. This pleasant property permits the author to prove a theorem about $g(z)$ which is analytic on $|z|=1$, and by taking the limit as $r \rightarrow 1^{-}$, obtain the same result about functions $f(z)$ in the same set when $f(z)$ is not analytic on $|z|=1$.

Unfortunately, the set $C V\left(R_{1}, R_{2}\right)$ does not behave quite as desired. If $f(z) \in$ $C V\left(R_{1}, R_{2}\right)$ and $r$ is fixed in $(0,1)$ it is possible that $g(z) \equiv f(r z) / r$ is not in $C V\left(R_{1}, R_{2}\right)$. However, one can prove that as $r \rightarrow 1^{-}$, the change in $R_{1}$ and $R_{2}$ is negligible. Thus (omitting a few details) we can always prove a theorem about $C_{r}=f(|z|=r)$ and then take the limit as $r \rightarrow 1^{-}$. Hence, without loss of generality, we may assume that $f(z)$ is analytic in $|z| \leqslant 1$. 
2. A coefficient bound. The function

$$
F(z) \equiv \frac{z}{1-A z}=z+\sum_{n=2}^{\infty} A^{n-1} z^{n}, \quad 0 \leqslant A<1,
$$

maps $E$ conformally onto the disk with center $A /\left(1-A^{2}\right)$ and radius $1 /\left(1-A^{2}\right)$. Hence $F(z) \in C V\left(R_{1}, R_{2}\right)$ where $R_{2}=1 /\left(1-A^{2}\right)$ and $R_{1}$ is any number in $\left(0, R_{2}\right]$. On the other hand, if $f(z) \in C V\left(R_{1}, R_{2}\right)$ then $f(E)$ is contained in some disk of radius $R_{2}$ (see [2]). An area theorem [1, vol. I, p. 27] gives

THEOREM 1. If $f(z) \in C V\left(R_{1}, R_{2}\right)$, then

$$
1+\sum_{n=2}^{\infty} n\left|a_{n}\right|^{2} \leqslant R_{2}^{2},
$$

and for each $k \geqslant 2$,

$$
\left|a_{k}\right| \leqslant\left(\left(R_{2}^{2}-1\right) / k\right)^{1 / 2}<R_{2} / k^{1 / 2} .
$$

The first inequality is sharp for each pair with $0 \leqslant R_{1} \leqslant R_{2}$.

From (2.2) we see that $R_{2} \geqslant 1$, and the set $C V\left(R_{1}, 1\right)$ contains only one member, $f(z) \equiv z$. The example function (2.1) suggests the conjecture that for all $k$ and $R_{2}$ and $f(z)$ in $C V\left(R_{1}, R_{2}\right)$,

$$
\left|a_{k}\right| \leqslant A^{k-1} \equiv\left(1-1 / R_{2}\right)^{(k-1) / 2}, \quad R_{2} \geqslant 1 .
$$

If (2.4) were true, it would be sharp, and thus a great improvement over (2.3). Now (2.4) may be the true bound for some values of $k$ and $R_{2}$, but the following example shọws that (2.4) cannot be correct for all $k \geqslant 2$ and $R_{2}>1$.

Set

$$
G(z)=z+a z^{k}, \quad a \geqslant 0, k \geqslant 2 .
$$

It is well known that $G(z)$ is convex if and only if $0 \leqslant a k^{2} \leqslant 1$. A moderate computation shows that $G(z) \in C V\left(R_{1}, R_{2}\right)$ where

$$
R_{2}=(1-k a)^{2} /\left(1-k^{2} a\right), \quad 0 \leqslant a k^{2}<1 .
$$

The value of $R_{1}$ is not needed in what follows. For small values of $k$ we find that $a \leqslant\left(1-1 / R_{2}\right)^{(k-1) / 2}$. However if we set $a=1 / 1000$ and $k=17$ in (2.6), we find that $R_{2} \approx 1.359$. On the other hand, these values used in (2.4) give $A^{k-1} \approx$ $0.0000237<1 / 1000$. Hence $(2.4)$ cannot give the sharp bound for $k=17$ and $R_{2} \approx 1.359$.

3. Koebe domains. Let $d=\left|w_{0}\right|$ where $w_{0}$ is a point nearest the origin on $\partial f(E)$.

THEOREM 2. If $f(z) \in C V\left(R_{1}, R_{2}\right)$, then

$$
|f(z)| \leqslant 2 R_{2}-d, \quad z \in \bar{E} .
$$

Further,

$$
d \geqslant R_{2}-\left(R_{2}^{2}-R_{2}\right)^{1 / 2} \equiv R_{K},
$$

and hence $f(E)$ always covers the disk centered at the origin with radius $R_{K}$. Both inequalities are sharp. 
Proof. By a rotation, we may set $w_{0}=-d$. The line from the origin to $w_{0}$ is normal to $\partial f(E)$ at $w_{0}$. From [2] a disk of radius $R_{2}$ and center at $R_{2}-d$ will cover $f(E)$. This proves (3.1). Further $F(z)$ given by (2.1) shows that for each $R_{2} \geqslant 1$, the inequality (3.1) is sharp. For this function, $R_{2}=1 /\left(1-A^{2}\right)$ and $d$ is given by (3.2) with the equal sign.

Since the disk described above covers $f(E)$,

$$
f(z) \prec \frac{B z}{1-A z}
$$

where $B=\left(2 R_{2}-d\right) d / R_{2}$ and $A=\left(R_{2}-d\right) / R_{2}$. But $f^{\prime}(0)=1$, and hence $B \geqslant 1$, with equality if and only if $f(z)=z /(1-A z)$. A brief computation with $\left(2 R_{2}-d\right) d / R_{2} \geqslant 1$ will give (3.2). This same function $z /(1-A z)$ with suitable $A$, shows that (3.2) is sharp. By a rotation, the inequality (3.2) gives the disk $|z|<R_{K}$ as the Koebe domain for the set $C V\left(R_{1}, R_{2}\right)$ for each $R_{2} \geqslant 1$.

The inequalities (3.1) and (3.2) give the

COROllary. If $f(z) \in C V\left(R_{1}, R_{2}\right)$ and $1 / 2 \leqslant d \leqslant 1$, then

$$
R_{2} \geqslant \frac{d^{2}}{2 d-1} \geqslant 1
$$

and

$$
|f(z)| \leqslant R_{2}+\left(R_{2}^{2}-R_{2}\right)^{1 / 2}, \quad z \text { in } E .
$$

Both inequalities are sharp.

We next consider a subordination in the reverse direction of (3.3). If $w_{0}$ is a point of $\partial f(E)$ that is closest to the origin, we may set $w_{0}=-d$ by a suitable rotation. From [2] the domain $f(E)$ will contain the open disk with radius $R_{1}$ and center $R_{1}-d$. Then $B z /(1-A z) \prec f(z)$ where $B=\left(2 R_{1}-d\right) d / R_{1}$ and $A=$ $\left(R_{1}-d\right) / R_{1}$. The condition $B \leqslant 1$ will give

THEOREM 3. If $f(z) \in C V\left(R_{1}, R_{2}\right)$ and $R_{1} \geqslant 1$, then $d \leqslant R_{1}-\left(R_{1}^{2}-R_{1}\right)^{1 / 2}$ and

$$
R_{1} \leqslant \frac{d^{2}}{2 d-1}, \quad \frac{1}{2}<d \leqslant 1 .
$$

Both inequalities are sharp.

Together with (3.2) we have

$$
R_{2}-\left(R_{2}^{2}-R_{2}\right)^{1 / 2} \leqslant d \leqslant R_{1}-\left(R_{1}^{2}-R_{1}\right)^{1 / 2}
$$

when $R_{1} \geqslant 1$.

4. Convex functions of order $\alpha$. Does either of the sets $C V\left(R_{1}, R_{2}\right)$ and $C V(\alpha)$ contain the other?

THEOREM 4. If $R_{2}<\infty$, and $0 \leqslant \alpha<1$, then

$$
C V(\alpha) \not \subset C V\left(R_{1}, R_{2}\right) \text {. }
$$


Proof. The function

$$
f(z)=\frac{1-(1-z)^{2 \alpha-1}}{2 \alpha-1}
$$

is in $C V(\alpha)$ if $\alpha \neq 1 / 2$ and $0 \leqslant \alpha \leqslant 1$. The function

$$
f(z)=-\ln (1-z)
$$

is in $C V(1 / 2)$. If $0 \leqslant \alpha \leqslant 1 / 2$, then the above examples are unbounded in $E$ and hence cannot belong to $C V\left(R_{1}, R_{2}\right)$ for any finite $R_{2}$ (see Theorem 2 ).

If $1 / 2<\alpha<1$, and $z=e^{i \theta}$, a brief computation, using (1.4), gives

$$
\rho=2^{\alpha} / 2 \alpha(1-\cos \theta)^{1-\alpha} \text {. }
$$

Hence $\rho \rightarrow \infty$ as $\theta \rightarrow 0$.

THEOREM 5. If $f(z) \in C V\left(R_{1}, R_{2}\right)$ and $R_{2}<\infty$, then for some $\alpha$,

$$
C V\left(R_{1}, R_{2}\right) \subset C V(\alpha) \text {. }
$$

In fact, $\alpha>1 / 4 R_{2}$.

Proof. From (1.4) and the definition of $C V\left(R_{1}, R_{2}\right)$ it follows that on the boundary of $E$

$$
\frac{\min \left|z f^{\prime}(z)\right|}{\min \operatorname{Re} Q_{C V}(f)} \leqslant R_{2}
$$

or

$$
\operatorname{Re} Q_{C V}(f) \geqslant \frac{\min \left|z f^{\prime}(z)\right|}{R_{2}}, \quad z=e^{i \theta} .
$$

Since the left side of (4.7) is a harmonic function, a minimum on $\partial E$ will hold throughout $E$. It is well known that if $f(z) \in C V(\alpha)$, then

$$
\left|f^{\prime}(z)\right| \geqslant 1 /(1+r)^{2(1-\alpha)} \geqslant 4^{-(1-\alpha)} .
$$

Since a function in $C V\left(R_{1}, R_{2}\right)$ is also convex, we can use (4.8) with $\alpha=0$. Then (4.7) gives $\operatorname{Re} Q_{C V}(f) \geqslant 1 / 4 R_{2}$.

This procedure can be iterated. Now that $f(z)$ is in $C V(\alpha)$ with $\alpha=\alpha_{1}=1 / 4 R_{2}$, we can use this in (4.8) and (4.7) to generate an $\alpha_{2}$. In general, the sequence

$$
\alpha_{k+1}=\frac{1}{\left(4^{1-\alpha_{k}}\right) R_{2}}
$$

is a bounded increasing sequence that has a limit $\beta$. Then (4.5) holds with $\alpha \geqslant \beta$. However, it is clear that $\beta$ is not the best lower bound for $\alpha$ and hence the precise determination of $\beta$ as the root of $\beta=1 / 4^{(1-\beta)} R_{2}$ that lies in $(0,1)$ is not important. Using a series for $4^{-1+x}$ we can show that

$$
\alpha>\beta>\frac{1}{4 R_{2}}+\frac{\ln 4}{16 R_{2}^{2}} .
$$

With a suitable choice of $A$, the function $z /(1-A z)$ is an example that lies in $C V\left(R_{1}, R_{2}\right)$ and in $C V(\gamma)$, where

$$
\gamma=2 R_{2}-1-2\left(R_{2}^{2}-R_{2}\right)^{1 / 2}=\frac{1}{4 R_{2}}+\frac{1}{8 R_{2}^{2}}+\cdots
$$


It is reasonable to conjecture that $\gamma$ is the sharp (largest) value of $\alpha$ for which (4.5) is true.

5. Other questions. What are the sharp bounds for $\left|a_{k}\right|$ ? It seems as though variational formulas for other classes of univalent functions cannot be applied to the class $\overline{C V}\left(R_{1}, R_{2}\right)$ or $C V\left(R_{1}, R_{2}\right)$. Is there a "nice" variational formula for either of these two classes?

We can obtain an Alexander type theorem if we define a class $S T\left(R_{1}, R_{2}\right)$ of starlike functions of bounded type. Thus $F(z) \in S T\left(R_{1}, R_{2}\right)$ if and only if $F(z)=$ $z f^{\prime}(z)$ for some $f(z)$ in $C V\left(R_{1}, R_{2}\right)$. For such a function

$$
R_{1} \leqslant \frac{|F(z)|}{\operatorname{Re}\left(z F^{\prime}(z) / F(z)\right)} \leqslant R_{2}
$$

as $|z| \rightarrow 1$. Then each theorem about $C V\left(R_{1}, R_{2}\right)$ will yield a companion theorem for the class $S T\left(R_{1}, R_{2}\right)$. But what are the geometric properties of functions in $S T\left(R_{1}, R_{2}\right)$ ?

Finally, we might ask questions about a new class of normalized functions $F(z)$ for which

$$
R_{1} \leqslant \frac{\left|z F^{\prime}(z)\right|}{\operatorname{Re}\left(z F^{\prime}(z) / F(z)\right)} \leqslant R_{2}
$$

as $|z| \rightarrow 1$. For such functions $R_{1} \leqslant d s / d \Phi \leqslant R_{2}$ on $\partial F(E)$ where $\Phi=\arg F\left(e^{i \theta}\right)$ and $s$ is arc length. Here the geometric character of $F(E)$ is clear, but the relation of this class to the classes $C V\left(R_{1}, R_{2}\right)$ and $S T\left(R_{1}, R_{2}\right)$ is not.

\section{REFERENCES}

1. A. W. Goodman, Univalent functions, vols. I and II, Mariner, Tampa, Florida, 1983.

2. Convex curves of bounded type, University of Southern Florida Seminar Notes.

3. M. S. Robertson, On the theory of univalent functions, Ann. of Math. 37 (1936), 374-408.

4. E. Study, Konforme Abbildung Einfachzusammenhangender Bereiche, Teubner, Leipzig and Berlin, 1913.

Department of Mathematics, University of South Florida, TAMPa, Florida 33620 